\title{
CANONICAL FORMS AND PRINCIPAL SYSTEMS FOR GENERAL DISCONJUGATE EQUATIONS
}

\author{
BY \\ WILLIAM F. TRENCH
}

\begin{abstract}
It is shown that the disconjugate equation (1) $L x \equiv\left(1 / \beta_{n}\right)(d / d t)$ $\cdot\left(1 / \beta_{n-1}\right) \cdots(d / d t)\left(1 / \beta_{1}\right)(d / d t)\left(x / \beta_{0}\right)=0, a<t<b$, where $\beta_{i}>0$, and $(2) \beta_{i} \in C(a, b)$, can be written in essentially unique canonical forms so that $\int^{b} \beta_{i} d t=\infty\left(\int_{a} \beta_{i} d t=\infty\right)$ for $1 \leq i \leq n-1$. From this it follows easily that (1) has solutions $x_{1}, \ldots, x_{n}$ which are positive in $(a, b)$ near $b(a)$ and satisfy $\lim _{t \rightarrow b^{-}} x_{i}(t) / x_{j}(t)=0\left(\lim _{t \rightarrow a^{+}} x_{i}(t) / x_{j}(t)=\infty\right)$ for $1 \leq i<j \leq n$. Necessary and sufficient conditions are given for (1) to have solutions $y_{1}, \ldots, y_{n}$ such that $\lim _{t \rightarrow b^{-}} y_{i}(t) / y_{j}(t)=\lim _{t \rightarrow a^{+}} y_{j}(t) / y_{i}(t)=0$ for $1 \leq i<j \leq n$. Using different methods, P. Hartman, A. Yu. Levin and D. Willett have investigated the existence of fundamental systems for (1) with these properties under assumptions which imply the stronger condition $\left(2^{\prime}\right) \beta_{i} \in C^{(n-i)}(a, b)$.
\end{abstract}

1. Introduction. A linear differential equation

$$
L x \equiv x^{(n)}+f_{1}(t) x^{(n-1)}+\cdots+f_{n}(t) x=0
$$

is said to be normal on an interval $I$ if $f_{i} \in C(I), 1 \leq i \leq n$, and disconjugate on $I$ if it has no nontrivial solution with more than $n-1$ zeros (counting multiplicities) on $I$. Polya [6] showed that if (1.1) is normal and disconjugate on $[a, b)$, then it can be written as

$$
L x \equiv \frac{1}{\beta_{n}} \frac{d}{d t} \frac{1}{\beta_{n-1}} \cdots \frac{d}{d t} \frac{1}{\beta_{1}} \frac{d}{d t} \frac{x}{\beta_{0}}=0, \quad a<t<b,
$$

where

$$
\beta_{i} \in C^{(n-i)}(a, b), \quad \beta_{i}>0, \quad 0 \leq i \leq n .
$$

Recently, Levin [5, Corollary 2.1] showed that if $(1.1)$ is normal on $(a, b)$, then it is disconjugate there if and only if it has a representation (1.2) which satisfies (1.3). (Levin actually assumed $f_{1}, \ldots, f_{n}$ to be locally integrable, rather than continuous, in $(a, b)$.)

Hartman [2], [3], [4] and Levin [5] independently showed that a disconjugate equation has fundamental systems, which Hartman called principal systems and Levin called hierarchical fundamental systems, with properties not exhibited by fundamental systems of other linear equations. We say that solutions $x_{1}, \ldots, x_{n}$

Presented to the Society, January 26, 1973; received by the editors September 28, 1972 and, in revised form, November 22, 1972.

AMS (MOS) subject classifications (1970). Primary 34C10; Secondary 34C99.

Key words and phrases. Disconjugacy, principal systems. 
of (1.2) form a principal system for (1.2) on $(a, b]$ if they are positive on some interval $(d, b)$ and

$$
\lim _{t \rightarrow b^{-}} \frac{x_{i}(t)}{x_{j}(t)}=0, \quad 1 \leq i<j \leq n,
$$

or a principal system for $(1.2)$ on $[a, b)$ if they are positive on some interval $(a, c)$ and

$$
\lim _{i \rightarrow a^{+}} \frac{x_{i}(t)}{x_{j}(t)}=\infty, \quad 1 \leq i<j \leq n .
$$

If $\left\{x_{1}, \ldots, x_{n}\right\}$ is a principal system for (1.2) on both $(a, b]$ and $[a, b)$, we call it a fundamental principal system for (1.2) on $[a, b]$ (see Willett [8]).

Hartman [2], [3], [4], Levin [5] and Willett [7], [8] have demonstrated the existence of principal systems for (1.1). (The work of Hartman and Levin is also discussed by Coppel [1].) Willett [8] obtained necessary and sufficient conditions for (1.1) to have fundamental principal systems on $[a, b]$. These investigations depend on the properties of Wronskians formed from solutions of (1.1). We use a different approach which applies to a larger class of equations; namely, those of the form (1.2) with (1.3) replaced by

$$
\beta_{i} \in C(a, b), \quad \beta_{i}>0, \quad 0 \leq i \leq n .
$$

A function $x$ is a solution of such an equation if the derivatives indicated in (1.2) exist and satisfy (1.2). If we define

$$
L_{0} x=\frac{x}{\beta_{0}}, \quad L_{j} x=\frac{1}{\beta_{j}}\left(L_{j-1} x\right)^{\prime}, \quad 1 \leq j \leq n,
$$

then $L x=L_{n} x$; hence (1.2) is equivalent to the system

$$
\begin{aligned}
\xi_{j}^{\prime} & =\beta_{j+1} \xi_{j+1}, \quad 0 \leq j \leq n-2, \\
\xi_{n-1}^{\prime} & =0,
\end{aligned}
$$

with $\xi_{j}=L_{j} x$, and therefore the usual existence and uniqueness theorems for linear equations apply to (1.2).

We shall write the operator $L$ in (1.2) as

$$
L=\frac{1}{\beta_{n}} \frac{d}{d t} \frac{1}{\beta_{n-1}} \cdots \frac{d}{d t} \frac{1}{\beta_{1}} \frac{d}{d t} \frac{\dot{\beta}}{\beta_{0}},
$$

and denote the class of such operators that satisfy $(1.4)$ by $D(a, b)$. In $\$ 2$ we show that an operator $L$ in $D(a, b)$ can be written in certain canonical forms, and use this to prove that (1.2) has principal systems on $(a, b]$ and $[a, b)$. We also give necessary and sufficient conditions for (1.2) to have fundamental principal systems on $[a, b]$. 
Throughout this paper $c$ is a fixed point in $(a, b)$ and $n \geq 2$.

2. Canonical forms and principal systems. The following notation of Willett provides a convenient way to represent solutions of (1.2).

If $\alpha_{1}, \alpha_{2}, \ldots$ are continuous on $(a, b)$, define

$$
\begin{aligned}
I_{0} & =1 \\
I_{j}\left(t, s ; \alpha_{j}, \ldots, \alpha_{1}\right) & =\int_{s}^{t} \alpha_{j}(\lambda) I_{j-1}\left(\lambda, s ; \alpha_{j-1}, \ldots, \alpha_{1}\right) d \lambda, \quad j \geq 1 .
\end{aligned}
$$

The functions

$$
x_{j}(t)=\beta_{0}(t) I_{j-1}\left(t, c ; \beta_{1}, \ldots, \beta_{j-1}\right), \quad 1 \leq j \leq n,
$$

are solutions of (1.2), and repeated application of Rolle's theorem shows that a nontrivial linear combination of $x_{1}, \ldots, x_{k}(1 \leq k \leq n)$ cannot have more than $k-1$ zeros in $(a, b)$. This implies that $\left\{x_{1}, \ldots, x_{n}\right\}$ is a fundamental system for (1.2) and (1.2) is disconjugate on $(a, b)$.

L'Hospital's rule shows that (2.1) defines a principal system for (1.2) on $(a, b]$ if

$$
\int^{b} \beta_{j} d t=\infty, \quad 1 \leq j \leq n-1
$$

while the functions

$$
z_{j}(t)=(-1)^{n-j} \beta_{0}(t) I_{n-j}\left(t, c ; \beta_{1}, \ldots, \beta_{n-j}\right), \quad 1 \leq j \leq n,
$$

form a principal system for (1.2) on $[a, b)$ if

$$
\int_{a} \beta_{j} d t=\infty, \quad 1 \leq j \leq n-1 .
$$

However, a given representation of an operator in $D(a, b)$ may fail to satisfy either (2.2) or (2.4). For example, the operator defined by $L x=x^{\prime \prime \prime}-x^{\prime}$ is in $D(-\infty, \infty)$, and can be represented as

$$
L=e^{t} \frac{d}{d t} e^{-2 t} \frac{d}{d t} e^{t} \frac{d}{d t} \dot{\overline{1}},
$$

which satisfies neither (2.2) nor (2.4). Nevertheless, $L$ can be rewritten as

$$
L=e^{t} \frac{d}{d t} e^{-t} \frac{d}{d t} e^{-t} \frac{d}{d t} \frac{.}{e^{-t}},
$$

which satisfies (2.2), or as

$$
L=e^{-t} \frac{d}{d t} e^{t} \frac{d}{d t} e^{t} \frac{d}{d t} \dot{\bar{e}} \overline{e^{t}}
$$

which satisfies (2.4). 
Theorem 1. Any operator $L$ in $D(a, b)$ has a representation (1.6) which satisfies (2.2), and this requirement determines $\beta_{0}, \ldots, \beta_{n}$ up to positive multiplicative constants with product 1 . The same statement is true with (2.2) replaced by (2.4).

We shall say that $L$ is in canonical form $a t b$ if (2.2) holds, or in canonical form at $a$ if (2.4) holds.

It is convenient to prove Theorem 1 by means of a series of lemmas and corollaries, as follows.

Lemma 1. If

$$
M=\frac{1}{\xi_{2}} \frac{d}{d t} \frac{1}{\xi_{1}} \frac{d}{d t} \dot{\xi_{0}}
$$

is in $D(a, b)$, with $\int^{b} \xi_{1} d t<\infty$, then $M$ can be rewritten as

$$
M=\frac{1}{\eta_{2}} \frac{d}{d t} \frac{1}{\eta_{1}} \frac{d}{d t} \frac{\dot{\eta}}{\eta_{0}}
$$

so that $\int^{b} \eta_{1} d t=\infty$, with

$$
\eta_{0}=\xi_{0} \int_{t}^{b} \xi_{1} d \tau, \quad \eta_{1}=\xi_{1}\left(\int_{t}^{b} \xi_{1} d \tau\right)^{-2}
$$

and

$$
\eta_{2}=\xi_{2} \int_{t}^{b} \xi_{1} d \tau
$$

Proof. Straightforward evaluation of $M x$, with $M$ as defined by (2.6), shows that (2.5) and (2.6) define the same operator.

Lemma 2. If

$$
N=\frac{1}{\mu_{3}} \frac{d}{d t} \frac{1}{\mu_{2}} \frac{d}{d t} \frac{1}{\mu_{1}} \frac{d}{d t} \dot{\bar{\mu}}
$$

is in $D(a, b)$, with

$$
\int^{b} \mu_{1} d t=\infty \text { and } \int^{b} \mu_{2} d t<\infty
$$

then $N$ can be rewritten as

$$
N=\frac{1}{\nu_{3}} \frac{d}{d t} \frac{1}{\nu_{2}} \frac{d}{d t} \frac{1}{\nu_{1}} \frac{d}{d t} \dot{\nu_{0}}
$$

where the $\nu_{i}$ are in $C(a, b)$ and positive, and $\int^{b} \nu_{i} d t=\infty, i=1,2$.

Proof. By applying the device of Lemma 1 to $\mu_{2}$, we obtain

$$
N=\frac{1}{\tilde{\nu}_{3}} \frac{d}{d t} \frac{1}{\tilde{\nu}_{2}} \frac{d}{d t} \frac{1}{\tilde{\nu}_{1}} \frac{d}{d t} \dot{\tilde{\nu}_{0}},
$$


with

$$
\begin{aligned}
& \text { (a) } \tilde{\nu}_{0}=\mu_{0}, \\
& \text { (b) } \tilde{\nu}_{1}=\mu_{1} \int_{t}^{b} \mu_{2} d \tau, \\
& \text { (c) } \tilde{\nu}_{2}=\mu_{2}\left(\int_{t}^{b} \mu_{2} d \tau\right)^{-2}, \\
& \text { (d) } \tilde{\nu}_{3}=\mu_{3} \int_{t}^{b} \mu_{2} d \tau .
\end{aligned}
$$

Now $\int^{b} \tilde{\nu}_{2} d t=\infty$; hence, if $\int^{b} \tilde{\nu}_{1} d t=\infty$, then we are finished, with $\nu_{i}=\tilde{\nu}_{i}$, $0 \leq i \leq 3$. If $\int^{b} \tilde{\nu}_{1} d t<\infty$, we apply the device of Lemma 1 to $\tilde{\nu}_{1}$ in (2.9) to obtain (2.8) with

$$
\begin{array}{ll}
\nu_{0}=\tilde{\nu}_{0} \int_{t}^{b} \tilde{\nu}_{1} d \tau, & \nu_{1}=\tilde{\nu}_{1}\left(\int_{t}^{b} \tilde{\nu}_{1} d \tau\right)^{-2}, \\
\nu_{2}=\tilde{\nu}_{2} \int_{t}^{b} \tilde{\nu}_{1} d \tau, & \nu_{3}=\tilde{\nu}_{3} .
\end{array}
$$

Now $\int^{b} \nu_{1} d t=\infty$. Let $a<t_{0}<t_{1}<b$; then integration by parts of

$$
\int_{t_{0}}^{t_{1}} \nu_{2} d t=\int_{t_{0}}^{t_{1}} \tilde{\nu}_{2}(t)\left(\int_{t}^{b} \tilde{\nu}_{1}(\tau) d \tau\right) d t
$$

in conjunction with $(2.10(\mathrm{~b}))$ and $(2.10(\mathrm{c}))$, yields

$$
\int_{t_{0}}^{t_{1}} \nu_{2} d t=\left.\left(\int_{t}^{b} \mu_{2} d \tau\right)^{-1}\left(\int_{t}^{b} \tilde{\nu}_{1} d \tau\right)\right|_{t_{0}} ^{t_{1}}+\int_{t_{0}}^{t_{1}} \mu_{1} d t .
$$

Therefore, (2.7) implies that $\int^{b} \nu_{2} d t=\infty$. This completes the proof of Lemma 2.

Lemmas 1 and 2 also hold with $\int^{b}$ and $\int_{t}^{b}$ replaced everywhere by $\int_{a}$ and $\int_{a}^{t}$. With these same substitutions, the proof of the assertions in Theorem 1 concerning the point $a$ can be obtained from the proof of the assertions concerning $b$; therefore, we present only the latter.

Proof of Theorem 1 (existence). The proof is by induction. Lemmas 1 and 2 imply existence for $n=2$ and $n=3$. Suppose $n \geq 4$ and existence has been established for any $(n-1)$ st order operator in $D(a, b)$. Then any $n$th order operator in $D(a, b)$ can be written as

$$
L=\frac{1}{\alpha_{n}} \frac{d}{d t} \frac{1}{\alpha_{n-1}} \cdots \frac{d}{d t} \frac{1}{\alpha_{1}} \frac{d}{d t} \frac{\dot{\alpha}}{\alpha_{0}},
$$

with

$$
\int^{b} \alpha_{j} d t=\infty, \quad 1 \leq j \leq n-2
$$


If $\int^{b} \alpha_{n-1} d t=\infty$, then $L$ is already in canonical form at $b$ and there is nothing to prove. If $\int^{b} \alpha_{n-1} d t<\infty$, we construct a sequence of representations

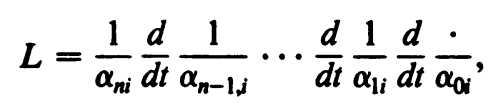

with $\alpha_{j 0}=\alpha_{j}, 0 \leq j \leq n$, and, for $i \geq 1$ :

(a) $\quad \alpha_{j i}=\alpha_{j, i-1}, \quad j \neq n-i+1, n-i$ or $n-i-1$,

(b) $\quad \alpha_{n-i+1, i}=\alpha_{n-i+1, i-1} \int_{t}^{b} \alpha_{n-i, i-1} d \tau$,

(c) $\quad \alpha_{n-i, i}=\alpha_{n-i, i-1}\left(\int_{t}^{b} \alpha_{n-i, j-1} d \tau\right)^{-2}$,

(d) $\quad \alpha_{n-i-1, i}=\alpha_{n-i-1, i-1} \int_{t}^{b} \alpha_{n-i, i-1} d \tau$.

This process terminates at the $i$ th step if

$$
\int^{b} \alpha_{j i} d t=\infty
$$

for all $j$ in $\{1, \ldots, n-1\}$. If $0 \leq i \leq n-2$, then (2.11), (2.12) and Lemma 2 imply that (2.13) holds for all such $j$ with the possible exception of $j=n-i-1$; hence, the process terminates when $i$ is such that $\int^{b} \alpha_{n-i-1, i} d t=\infty$, or when $i=n-1$, whichever occurs first. If it terminates with $i=r$, we take $\beta_{j}=\alpha_{j r}$ in (1.6). This completes the induction and the existence proof.

Coupled with the earlier discussion of (2.1) and (2.3), this existence theorem yields the following corollary.

Corollary 1. If $L$ is in $D(a, b)$, then (1.2) has principal systems on $(a, b]$ and $[a, b)$.

The essential uniqueness of the canonical forms is a consequence of the following lemma, which was stated without proof by Levin [5, p. 59] for disconjugate equations of the form (1.1). The lemma follows directly from the properties of principal systems.

Lemma 3. If $\left\{x_{1}, \ldots, x_{n}\right\}$ and $\left\{y_{1}, \ldots, y_{n}\right\}$ are principal systems for (1.2) on $(a, b]$ $([a, b))$, then

$$
y_{i}=\sum_{j=1}^{i} a_{i j} x_{j} \quad\left(y_{i}=\sum_{j=i}^{n} a_{i j} x_{j}\right)
$$

where the $\left\{a_{i j}\right\}$ are constants and $a_{i i}>0$.

Corollary 2. If $\left\{x_{1}, \ldots, x_{n}\right\}$ and $\left\{y_{1}, \ldots, y_{n}\right\}$ are fundamental principal systems for (1.2) on $[a, b]$, then $x_{i}=\lambda_{i} y_{i}, 1 \leq i \leq n$, where $\lambda_{1}, \ldots, \lambda_{n}$ are positive constants. 
Corollary 3. Let $L$ in (1.6) be in canonical form at $b$, suppose $L_{0}, \ldots, L_{n-1}$ are as defined by (1.5), and let $\left\{y_{1}, \ldots, y_{n}\right\}$ be a principal system for $(1.2)$ on $(a, b]$. Then:
(a)
$L_{i} y_{j}=0$,
$1 \leq j \leq i$
(b) $\quad L_{i} y_{i+1}=\rho_{i}(=$ positive constant $)$,
$0 \leq i \leq n-1$.

Proof. If $x_{i}$ is as defined by (2.1), then (2.14) holds with $\rho_{i}=1$. Hence, the conclusion follows from Lemma 3.

An analogous result is valid if $\left\{y_{1}, \ldots, y_{n}\right\}$ is a principal system for (1.2) on $[a, b)$.

Proof of Theorem 1 (essential uniqueness). Suppose (1.6) and

$$
L=\frac{1}{\alpha_{n}} \frac{d}{d t} \frac{1}{\alpha_{n-1}} \cdots \frac{d}{d t} \frac{1}{\alpha_{1}} \frac{d}{d t} \frac{\dot{\alpha}}{\alpha_{0}}
$$

are both canonical forms for $L$ at $b$. Then

$$
y_{j}(t)=\alpha_{0}(t) I_{j-1}\left(t, c ; \alpha_{1}, \ldots, \alpha_{j-1}\right), \quad 1 \leq j \leq n,
$$

defines a principal system for (1.2) on $(a, b]$, and (2.14b) yields

$$
L_{j} \alpha_{0}(t) I_{j}\left(t, c ; \alpha_{1}, \ldots, \alpha_{j}\right)=\rho_{j}, \quad 0 \leq j \leq n-1,
$$

with $L_{0}, \ldots, L_{n-1}$ as defined by (1.5). Suppose it is known that

$$
\alpha_{0}=\rho_{0} \beta_{0}, \quad \alpha_{1}=\left(\rho_{1} / \rho_{0}\right) \beta_{1}, \ldots, \alpha_{j-1}=\left(\rho_{j-1} / \rho_{j-2}\right) \beta_{j-1},
$$

for some $j, 2 \leq j \leq n-1$. (Direct computation with (2.16) verifies (2.17) for $j=2$.) Then, from (2.16),

$$
\rho_{j}=\rho_{j-1} L_{j} \beta_{0}(t) I_{j}\left(t, c ; \beta_{1}, \ldots, \beta_{j-1}, \alpha_{j}\right)=\rho_{j-1} \alpha_{j} / \beta_{j},
$$

which yields (2.17) with $j$ replaced by $j+1$. Thus, by induction, (2.17) holds for $j=n$, and (2.15) can be rewritten as

$$
L=\frac{1}{\rho_{n-1}} \frac{1}{\alpha_{n}} \frac{d}{d t} \frac{1}{\beta_{n-1}} \cdots \frac{d}{d t} \frac{1}{\beta_{1}} \frac{d}{d t} \dot{\beta_{0}} .
$$

Now define

$$
x_{n+1}=\beta_{0}(t) I_{n}\left(t, c ; \beta_{1}, \ldots, \beta_{n}\right) .
$$

From (1.2), $L x_{n+1}=1$, while from (2.18), $L x_{n+1}=\left(1 / \rho_{n-1}\right) \beta_{n} / \alpha_{n}$; hence $\alpha_{n}$ $=\beta_{n} / \rho_{n-1}$, which completes the proof.

Although (1.2) always has principal systems on $(a, b]$ and $[a, b)$, the next theorem shows that it may fail to have a fundamental principal system on $[a, b]$. 
Theorem 2. Suppose the operator $L$ in (1.6) is in canonical form at $b$. Then (1.2) has a fundamental principal system on $[a, b]$ if and only if

$$
\int_{a} \beta_{i} d t<\infty, \quad 1 \leq i \leq n-1
$$

Proof. For sufficiency, observe that (2.19) implies that the integrals in

$$
x_{j}(t)=\beta_{0}(t) I_{j-1}\left(t, a ; \beta_{1}, \ldots, \beta_{j-1}\right), \quad a<t<b,
$$

exist for $1 \leq j \leq n$, and that these functions form a fundamental system for (1.2) on $[a, b]$. For necessity, suppose $\left\{y_{1}, \ldots, y_{n}\right\}$ is a fundamental principal system for (1.2) on $[a, b]$. From Lemma 3 ,

$$
y_{i}(t)=\beta_{0}(t) \sum_{j=1}^{i} a_{i j} I_{j-1}\left(t, c ; \beta_{1}, \ldots, \beta_{j-1}\right), \quad 1 \leq i \leq n,
$$

for suitable constants $\left\{a_{i j}\right\}$. In particular, $y_{1}=a_{11} \beta_{0}$, and therefore

$$
0=\lim _{t \rightarrow a^{+}} \frac{y_{2}(t)}{y_{1}(t)}=a_{11}^{-1} \lim _{t \rightarrow a^{+}}\left(a_{21}+a_{22} I_{1}\left(t, c ; \beta_{1}\right)\right) .
$$

Since $a_{22} \neq 0$, this implies that $I_{1}\left(a, c ; \beta_{1}\right)$ exists, which confirms $(2.19)$ for $j=1$, and completes the proof if $n=2$. Now suppose $n>2$ and the desired conclusion holds for all operators in $D(a, b)$ of order $n-1$. Since (2.14(a)) implies that $\left\{y_{1}, \ldots, y_{n-1}\right\}$ is a fundamental principal system on $[a, b]$ for the equation

$$
L_{n-1} x \equiv \frac{1}{\beta_{n-1}} \frac{d}{d t} \frac{1}{\beta_{n-2}} \cdots \frac{d}{d t} \frac{1}{\beta_{1}} \frac{d}{d t} \frac{x}{\beta_{0}}=0,
$$

it follows from the induction assumption that (2.19) holds with $n$ replaced by $n-1$ and therefore that $x_{1}, \ldots, x_{n-1}$, as defined for $1 \leq j \leq n-1$ by (2.20), also form a fundamental principal system for $(2.22)$ on $[a, b]$. Therefore, from Corollary 2,

$$
y_{n-1}(t)=\lambda_{n-1}^{-1} \beta_{0}(t) I_{n-2}\left(t, a ; \beta_{1}, \ldots, \beta_{n-2}\right) .
$$

This, together with (2.21) and repeated application of L'Hospital's rule, yields

$$
0=\lim _{t \rightarrow a^{+}} \frac{y_{n}(t)}{y_{n-1}(t)}=\lambda_{n-1} \lim _{t \rightarrow a^{+}}\left(a_{n, n-1}+a_{n n} I_{1}\left(t, c ; \beta_{n-1}\right)\right),
$$

which implies that $I_{1}\left(a, c ; \beta_{n-1}\right)$ exists. This confirms (2.19) and completes the induction. 
It can be shown in the same way that if (1.6) is in canonical form at $a$, then (1.2) has a fundamental principal system on $[a, b]$ if and only if

$$
\int^{b} \beta_{i} d t<\infty, \quad 1 \leq i \leq n-1 .
$$

\section{REFERENCES}

1. W. A. Coppel, Disconjugacy, Lecture Notes in Math., vol. 220, Springer-Verlag, Berlin, 1971.

2. P. Hartman, Disconjugate nth order differential equations and principal solutions, Bull. Amer. Math. Soc. 74 (1968), 125-129. MR 36 \#5440.

3. - Principal solutions of disconjugate n-th order linear differential equations, Amer. J. Math. 91 (1969), 306-362. MR 40 \#450.

4.—, Corrigendum and addendum: Principal solutions of disconjugate n-th order linear differential equations, Amer. J. Math. 93 (1971), 439-451. MR 45 \#648.

5. A. Ju. Levin, Non-oscillation of solutions of the equation $x^{(n)}+p_{1}(t) x^{(n-1)}+\cdots+p_{n}(t) x=0$, Uspehi Mat. Nauk 24 (1969), no. 2 (146), 43-96 = Russian Math. Surveys 24 (1969), no. 2, 43-99. MR 40 \# 7537.

6. G. Polya, On the mean-value theorem corresponding to a given linear homogeneous differential equation, Trans. Amer. Math. Soc. 24 (1924), 312-324.

7. D. Willett, Asymptotic behaviour of disconjugate nth order differential equations, Canad. J. Math. 23 (1971), 293-314. MR 45 \#2275.

8. - Disconjugacy tests for singular linear differential equations, SIAM J. Math. Anal. 2 (1971), $536-545$.

Department of Mathematics, Drexel University, Philadelphia, Pennsylvanin 19104 